\title{
Strange lesions after stem-cell therapy
}

In a stark reminder that stem-cell therapy is uncharted territory, a stem-cell transplant given to a patient in Thailand who had kidney disease resulted in the development of cellular masses not previously reported. The lesions, described in a paper published online on 17 June in the Journal of the American Society of Nephrology, were not directly linked to the patient's subsequent death (D. Thirabanjasak et al. J. Am. Soc. Nephrol. doi:10.1681/ ASN.2009111156; 2010).

With hundreds of poorly regulated clinics that offer unproven stem-cell therapies now running, notably in China and Thailand, the episode is a warning to patients who may be considering such treatment.

The patient had lupus nephritis, in which the immune system attacks the kidneys. In 2006, she underwent a procedure at a private clinic in which her own haematopoietic stem cells - which can develop into any type of blood cell - were injected into her kidneys. Details of the clinic and the rationale behind the treatment have not been released.

Haematopoietic stem cells have been used to treat lupus nephritis with some reports of success. But they are usually injected into the bloodstream, not the kidney, in an attempt to 'reset' the immune system.

\section{"She didn't get any better from stem cells, we can say that."}

Six months later, the patient complained of pain and blood in the urine. Imaging studies revealed a four-centimetre mass on her left kidney and smaller masses in the kidney, liver and adrenal gland. Doctors at Chulalongkorn University in Bangkok removed the kidney, believing a malignant tumour to be present. But further analysis showed that it was something else.

"I had never seen anything like it," says Paul Thorner, a pathologist at the Hospital for Sick Children in Toronto, Canada, who has a joint position at Chulalongkorn University and was a co-author on the paper. Thorner coined a term - angiomyeloproliferative - to describe the proliferation of blood-vessel and bone-marrow cells the team found.

Patients are undergoing other experimental stem-cell therapies, but there is usually little follow-up to establish safety or efficacy. In one case reported last year, a boy treated with fetal stem cells at a Russian clinic developed tumours in his brain and spinal cord. In the Thai case, no post-mortem was carried out, as far as Thorner is aware, so his team could work only on the removed kidney. It is not known whether the smaller masses were linked to the main mass, or what would have happened had the patient not died from other complications. "She didn't get any better from stem cells, we can say that," says Thorner, who may try to reproduce the masses in animal experiments.

David Cyranoski 